\title{
Multiple Endocrine Neoplasia Type 1: A Chinese Family with a New Germline Mutation(c.201delC) and Detection Errors Due to an Intron Mutation
}

\section{Fan Zhang}

China Medical University First Hospital

\section{Xinyue Lin}

China Medical University First Hospital

\section{Xiaoli Wang}

China Medical University First Hospital

Xiaohui Yu ( $\square$ yuxh_1978@126.com )

China Medical University First Hospital https://orcid.org/0000-0003-4802-9673

\section{Research article}

Keywords: Multiple Endocrine Neoplasia type 1, MEN1, germline mutation, Whole exon sequencing, exon

Posted Date: October 26th, 2020

DOI: https://doi.org/10.21203/rs.3.rs-41525/v3

License: (c) (i) This work is licensed under a Creative Commons Attribution 4.0 International License. Read Full License 


\section{Abstract}

Background: Multiple endocrine neoplasia type 1 (MEN1) is a hereditary cancer syndrome caused by germline mutations in the MEN1 gene located on chromosome 11q13. The three main endocrine tissues affected most frequently by tumors in MEN1 are the parathyroid (95\%), enteropancreatic neuroendocrine tissues (50\%), and anterior pituitary (40\%). The purpose of this study was to report on a Chinese family with a new heterozygous MEN1 mutation in exon 2 [NM_130802: c.201delC (p.Ala68Profs*51) with localization to Chr11:64577381 on assembly GRCh37]. Methods: A 49-year-old female patient (proband) was admitted to the hospital due to intermittent recurrent hypoglycemia symptoms. After a series of examinations, we found that she had a pancreatic tumor, parathyroid tumor, adrenal tumor, and suspicious gastrinoma. Due to the proband's suspicious clinical manifestations of MEN1, we performed genetic detection for the proband and her immediate family members. We also used multiplex ligationdependent probe amplification (MLPA) to rule out homozygous mutations caused by a deletion of gene fragments in the proband and her immediate family members. Results: A new MEN1 germline mutation [c.201 delC (p.Ala68Profs ${ }^{\star 51)], ~ w h i c h ~ c o u l d ~ i n d u c e ~ M E N 1, ~ w a s ~ f o u n d ~ b y ~ t h e ~ g e n e t i c ~ t e s t . ~ T w o ~ o f ~ t h e ~}$ proband's six relatives were found by the genetic test to have the same mutations as the proband, and the proband's sister also had the typical symptoms of MEN1. However, the first and second genetic detection results showed that they were homozygous mutations, which did not conform to Mendelian inheritance laws. The MLPA results showed that the gene deletion was absent in the MEN1. The results from the third genetic detection (redesigned the primer) showed that they had a heterozygous mutation. The detection errors might due to an intron mutation. Conclusions: A new germline mutation, which can improve the identification of clinical phenotypes and the early diagnosis of MEN1, was identified. An intron mutation may lead to incorrect results of homozygous, which is incompatible with Mendelian genetic law. Redesigning the primers for gene detection could avoid this situation.

\section{Background}

Multiple endocrine neoplasia type 1 (MEN1), characterized by neoplasia in multiple endocrine and nonendocrine tissues, is an autosomal dominant hereditary tumor syndrome with a prevalence of about $1 / 30,000$. The three major endocrine tissues affected by tumors in MEN1 are the parathyroid (95\%), enteropancreatic neuroendocrine (50\%), and anterior pituitary (40\%). A practical definition of MEN1 is the presence of two of the three main MEN1-related endocrine tumors (parathyroid adenomas, enteropancreatic endocrine tumors, and pituitary tumors) (1). In addition, MEN1 patients could present with many other hormone-secreting, hormone nonsecreting, and nonendocrine tumors, such as adrenal cortical tumors, foregut carcinoids (bronchial, thymic or of the gastric enterochromaffin-like cells), facial angiofibromas, truncal collagenomas, lipomas, meningiomas, Barrett's esophagus, leiomyoma (uterine in females or in the esophagus), and ependymoma (1). Approximately 25\% of MEN1 patients die of malignant gastrinomas (gastrointestinal neuroendocrine tumors) or foregut carcinoid tumors (1).

The related gene MEN1, which encodes the protein MENIN $(2,3)$, was first reported in 1988 (4) and is located on chromosome region $11 q 13(2,3)$. It is composed of 10 exons that encode a 610 -amino acid 
nuclear protein. MENIN interacts with the activator protein 1 (AP1) transcription factor JunD to inhibit JunD-activated transcription (5), which is related to cell growth regulation, cell cycle, genome stability, and synaptic plasticity. Analysis of the MEN1 gene mutation is helpful for distinguishing MEN1 syndrome from other sporadic tumors, such as somatic loss of heterozygosity (LOH) on chromosome 11q13, which is observed in $5-50 \%$ of such non-hereditary common sporadic tumors (6). MEN1 gene mutations are distributed throughout the coding region, which has no obvious hot spots and no obvious genotype/phenotype correlation with tumor spectrum or clinical characteristics. More than 1,000 mutations have been reported, including frameshift deletions or insertions $(40 \%)$, nonsense $(20 \%)$ and missense $(25 \%)$ mutations, splice site changes (8\%), deletions or insertions (6\%), and large deletions (1\%) (6). Thus, $70-75 \%$ of the MEN1 gene mutations are inactivating, causing premature protein truncation. However, many mutations (such as rare missense in nonfamilial cases) can be classified as mutations of unknown clinical significance due to the lack of available assays that could provide evidence for an adverse physiological consequence of such mutations (7).

Whole exon sequencing (WES) was performed on the proband in this study, who had a pancreatic tumor, parathyroid tumor, adrenal tumor, and a suspicious gastrinoma. The suspected MEN1 mutation site in exon 2 was screened using a combination of bioinformatics and Sanger sequencing. The results indicated that a mutation in an intron affected the sequencing results and led to the occurrence, at first, of homozygotes. All patients were found to be heterozygotes by resequencing. A new MEN1 germline mutation [NM_130802: c.201delC (p.Ala68Profs*51) on Chr11:64577381 on assembly GRCh37] was found, which was the genetic pathogenesis of MEN1 in this family.

\section{Methods}

\section{Patients}

The proband (II-3) was a 49-year-old woman who was born to a family native to Liaozhong, Liaoning Province of China. She was admitted to our hospital with a "3-year duration of large appetite and rapid hungering, intermittent recurrent fatigue, and palpitations, sweating for 2 years." A series of examinations were performed, and the main diagnosis of the proband was MEN1. She had pancreatic insulinoma, a tumor of the adrenal gland, primary hyperparathyroidism (adenoma of the parathyroid), hyperprolactinemia, gastric and duodenal ulcers (possibly Zollinger-Ellison syndrome), a right renal cyst, and myoma of the uterus.

It is well known that MEN1 is an autosomal dominant genetic disease. Therefore, in addition to the proband, we also carried out genetic tests on her relatives. The patient's family consisted of three generations, including ten members. The proband's father (I-2) of the first generation was deceased. Seven people were tested. Three members carried gene mutations, including the proband (II-3), the proband's sister (II-1), and the proband's niece (III-1). The results of the other relatives' genetic tests indicated normal MEN1 gene sequences. No close relatives among the members of this family had 
married. The genetic map of the family members is given in Fig. $1 \mathrm{~h}$. All family members in this study provided signed informed consent.

\section{Laboratory examination}

Serum tests for pituitary-related hormones, thyroid and parathyroid hormones, blood glucose, insulin, Cpeptide, and bone metabolism-related tests were accomplished in the proband, as were the related gland function evaluation test.

\section{Imaging examination}

For a diagnosis of MEN1, the proband completed several imaging examinations, including an ultrasound, CT (computed tomography), MRI (magnetic resonance imaging), endoscopic ultrasound (EUS), and parathyroid ECT (emission computed tomography).

\section{Whole exome sequencing (WES)}

A 5-ml sample of peripheral blood was collected from the proband and each of her six relatives mentioned, anticoagulated with ethylenediaminetetraacetic acid (EDTA), and stored at $-80^{\circ} \mathrm{C}$. Later, whole-genome DNA was extracted. The proband's DNA samples were sequenced at different depths. An approx. $900 \mathrm{bp}\left(2^{\text {nd }}, 800 \mathrm{bp} ; 3^{\text {rd }}, 170 \mathrm{bp}\right)$ insert size sequencing library was constructed from $2 \mu \mathrm{g}$ genomic DNA using the TruSeq DNA sample preparation kit (Illumina, USA), and then sequenced on an Illumina HiSeq sequencing platform and the whole exon capture platform of the Roche probe group. The sequencing depth and coverage of each sample met the requirements for a bioinformatics data analysis. Exons were captured using PCR amplification technology and Sanger sequencing by ABI3730. The sequencing results were obtained by the DNASTAR subroutine SeqMan software. The suspected pathogenic mutations were classified according to the ACMG (American College of Medical Genetics and Genomics) gene mutation classification system, and unreported mutations or clinical ambiguous mutations were predicted by the software and annotated by the population database. Sanger sequencing was performed on the peripheral blood samples of the six family members using an $A B I 3730$ sequencer to verify the co-segregation of genotype and phenotype. The DNA sequence of MEN1 (NM_130802) was queried in the Ensembl Genome Browser, and Primer Premier 6.0 was used to design primers for verification:

MEN1-Exon2-1F; 5'-GGGTGGAACCTTAGCGG-3', MEN1-Exon2-1R; 5'- TGCAGCCCAATTTCATCT-3'. MEN1-Exon2-2F; 5'-GGGTGGAACCTTAGCGG-3', MEN1-Exon2-2R; 5'- GTTTCCCAACTGCAAAGAGG-3'. MEN1-Exon2-3F; 5'-GACCTGGTGCTCCTTTCCTT-3', MEN1-Exon2-3R; 5'- GGGCGGCGATGATAGACA-3'. Multiplex ligation-dependent probe amplification (MLPA) 
DNA (20-500 ng) was denatured by heating to $98^{\circ} \mathrm{C}$ in a thermocycler. The multiplex ligation-dependent probe amplification (MLPA) probes and buffer were added, and then the samples were left overnight at 60 ${ }^{\circ} \mathrm{C}$ for hybridization. The ligase and ligase buffer were added, and the samples were ligated at $54{ }^{\circ} \mathrm{C}$ for $15 \mathrm{~min}$. The ligase was inactivated by heating to $98^{\circ} \mathrm{C}$. PCR primers, dNTPs, and polymerase were added, and the PCR was started. The products were analyzed by capillary electrophoresis.

\section{Results}

\section{Pancreas-related results}

Islet cell tumors are the most common endocrine gland tumor in MEN1. Several symptoms (large appetite and rapid hungering, intermittent recurrent fatigue, palpitations, and sweating) in the proband suggested that pancreatic insulinoma had greater likelihood. In addition, during hospitalization, the proband experienced hypoglycemia symptoms twice. Meanwhile, the proband's serum glucose, insulin (INI), Cpeptide (CPS), and pro-insulin (pro-INS) levels were tested, and the results showed that glucose was decreased while the insulin level was either in the normal reference range or increased at the time of the hypoglycemia (Table 1). The insulin release indexes were 0.55 and 0.52 (normal range $<0.3$ ) during the twice hypoglycemia, respectively. A prolonged oral glucose tolerance test (OGTT) was performed, and the results presented hypoglycemia at the $240^{\text {th }}$ and $300^{\text {th }}$ minute with glucose levels of $2.01 \mathrm{mmol} / \mathrm{L}$ and $1.95 \mathrm{mmol} / \mathrm{L}$, respectively. The corresponding insulin release indexes were 0.48 and 0.43 , respectively (Table 2).

The imaging examinations provided us with more information. The CT results of the pancreas showed that the mass in the pancreatic neck $(8.0 \times 6.8 \times 8.4 \mathrm{~cm})$ could have been a solid pseudopapillary tumor (Fig. 1a1-a4). Circular reinforced nodules in the tail of the pancreas $(2.8 \times 2.7 \mathrm{~cm})$ (Fig. 1b1-b4) were considered to be homologous with the pancreatic neck mass (solid pseudopapillary tumor). The arterial phase of the pancreatic tail artery significantly strengthened the small nodules at the early stage, which could have been a neuroendocrine tumor. EUS is considered to be the most sensitive single method for the detection of pancreatoduodenal tumors. Compared with a CT scan and somatostatin receptor imaging, EUS has obvious advantages (8). The patient's ultrasound gastroscopy showed that the head of the pancreas could not be observed in the full field because the mass was too big (Fig. 1c1-c2). The mass in the tail of the pancreas $(2.81 \times 2.02 \mathrm{~cm})(\mathrm{Fig} .1 \mathrm{c} 3-\mathrm{c} 4)$ was a hypoechoic mass, and the internal echoes were mixed.

Therefore, the mass in the pancreas was likely an islet cell tumor. Later on, we checked other endocrine glands of the proband.

\section{Parathyroid-related results}

In MEN1 patients, the prevalence of parathyroid tumors is about $95 \%$ (7). The proband's serum tests showed that the serum calcium $\left(\mathrm{Ca}^{2+}\right)$ was increased $(2.47-2.74 \mathrm{mmol} / \mathrm{L}$, normal range $2.17-2.57$ 
$\mathrm{mmol} / \mathrm{L})$ and serum phosphorus $(\mathrm{P})$ was decreased $(0.71-0.96 \mathrm{mmol} / \mathrm{L}$, normal range $0.81-1.52$ $\mathrm{mmol} / \mathrm{L}$ ). The serum parathyroid hormone was $553.6 \mathrm{pg} / \mathrm{mL}$ (normal range $15-65 \mathrm{pg} / \mathrm{mL}$ ).

The results of the patient's bone mineral density measurements presented a decrease in bone mass in the lumbar spine and hip joint bones. Imaging modalities, such as parathyroid ultrasound and ECT, are valuable in MEN1 patients (9). Parathyroid ultrasound showed a low echo at the posterior margin of the thyroid (right, $7.9 \times 4.5 \mathrm{~mm}$; left, $11.0 \times 3.6 \mathrm{~mm}$ ). Fifteen minutes after the $99 \mathrm{mTc}$-stabimidine (MIBI) injection, the parathyroid ECT demonstrated an area of increased imaging agent in the lower lobe of the left thyroid (Fig. 1d1, d3). Two hours after the injection, the imaging agent that was increased in the left lobe of the thyroid had not significantly decreased (Fig. 1d2, d4). This phenomenon was caused by a highly functional parathyroid adenoma.

The above results confirmed that the proband had primary hyperparathyroidism that could derive from the highly functional parathyroid adenoma.

\section{Adrenal gland-related results}

Asymptomatic adrenal tumors occur in about $20 \%$ to $73 \%$ of MEN1 patients. Patients with MEN1 tend to develop aldosterone and adrenocortical cancer but are less likely to develop pheochromocytoma than those with adrenal incidentaloma (10). The proband's CT result showed that a mass occupied the right adrenal gland (Fig. 1e).

The biochemical evaluation of adrenal tumors includes an evaluation of plasma free cortisone (COR), evening cortisol, plasma renin, and aldosterone concentration, and a 1-mg overnight dexamethasone (DXM) suppression test (9). In this case, the basal COR rhythm was turbulent. The 1-mg overnight DXM suppression test results demonstrated that the inhibition rate of COR was $76.4 \%$ (normal range $>50 \%$ ). Therefore, Cushing syndrome was excluded. The plasma renin and aldosterone concentrations were normal. Moreover, the patient did not have hypertension.

All of these findings suggested that a nonfunctional adrenal tumor existed.

\section{Pituitary-related results}

The serum prolactin (PRL) was two times the normal upper limit (1253 mIU/L, normal range 40-530 $\mathrm{mIU} / \mathrm{L}$; rechecked $1395 \mathrm{mIU} / \mathrm{L}$ ). Serial basal PRL levels greater than $4240 \mathrm{mIU} / \mathrm{L}$ confirmed a prolactinoma (9). The results of the metoclopramide stimulation test showed that PRL was 1.88 times higher than the basic value and the inhibition rate of PRL was $60.7 \%$ (normal range $>50 \%$ ), as found by the bromocriptine inhibition test. This indicated that the patient suffered from a prolactinoma of the pituitary. Other pituitary hormones, including growth hormone (GH), IGF-1 (insulin-like growth factor 1), luteinizing hormone (LH), follicle-stimulating hormone ( $\mathrm{FSH})$, thyroid-stimulating hormone (TSH), and free-thyroxine (FT4), were normal. 
An MRI of the pituitary gland with contrast is the best diagnostic imaging (9). The pituitary-enhanced MRI examination showed that the hypophysis was full in shape (height $0.95 \mathrm{~cm}$ ) (Fig. 1f1-f3). Based on the above serum and imaging examination, the proband could have had a prolactinoma. The small size of the pituitary tumor could have led to the inconspicuous imaging evidence.

\section{Stomach and duodenum-related tumor}

The incidence of neuroendocrine tumors (NETs) in gastrointestinal and pancreatic tissues is about $30 \%$ to $75 \%$. However, autopsy studies have shown that the prevalence is as high as $80 \%$ to $100 \%(11)$. NETs without function are more common than functional tumors. Moreover, because approximately $25 \%$ of MEN1 patients die from cancer due to malignant gastrinomas (1), an examination of the gastroduodenum is necessary. Serum pepsinogen I and gastrin 17 (GAS-17) were increased significantly $(547 \mu \mathrm{g} / \mathrm{L}$, normal range $70-150 \mu \mathrm{g} / \mathrm{L} ; 78.6 \mathrm{pmol} / \mathrm{L}$, normal range $0.01-5 \mathrm{pmol} / \mathrm{L}$, respectively). The antibody result for Helicobacter pylori infection was negative.

Gastroscopy showed multiple submucosal uplifts (the largest was about $0.6 \times 0.8 \mathrm{~cm}$ ) at the junction of the duodenal bulb and the descending part of the duodenum (Fig. 1g1). A distal shallow ulcer in the descending part of the duodenum was about $1.0 \times 0.5 \mathrm{~cm}$ in size (Fig. 1g2). The pathological diagnosis was moderate inflammation and erosion of the descending part of the duodenum. Duodenal gastrinomas in MEN1 syndrome are usually small (smaller than $1 \mathrm{~cm}$ ), multifocal, and occur mainly in the proximal duodenum (9). Therefore, no obvious gastrinoma lesions were found during the gastroscopy.

Known as Zollinger-Ellison syndrome (ZES), peptic ulcer disease can be caused by functioning NETsecreting gastrin because of increased gastric acid secretion (9). As a result of the increased level of gastrin 17 and the occurrence of duodenal ulcers, the proband was likely to have had gastrinomas.

\section{Whole exome sequencing}

In $90 \%$ of cases, MEN1 is usually inherited from the affected parent; the other $10 \%$ of cases occur by de novo mutation. Germline inactivating mutations of the MEN1 gene on chromosome 11 is the main cause of MEN1 (9). To determine whether the gene had changed, the proband and six relatives had exon sequencing and bioinformatics analysis.

The WES results of the first experiment (NM_130802-1F, NM_130802-1R) showed that the proband (II-3), her sister (II-1), and her niece (III-1) had c.201 delC change of NM_130802 in exon 2, which caused amino acid changes (p.Ala68Profs*51) that led to MEN1. Interestingly, the genetic changes were homozygous mutations (Fig. 2, MEN1-Exon2-1F, 1R). No genetic mutations were found in the other relatives. However, we analyzed the patient's family pedigree, and neither the mother (I-1) nor the son (III-2) of the proband had this genetic mutation. This result was not consistent with Mendelian genetic law. Therefore, we commissioned a technician to redesign a part of the primers (MEN1-Exon2-2R). We retested the samples and got the same result (c.201delC) (Fig. 2, MEN1-Exon2-2F, 2R). 
Beijers et al. (12) found a germline and somatic mosaicism in a family with MEN1. They analyzed the blood using MLPA, which showed a minimal but consistently decreased signal for the MEN1-specific MLPA probes. The results from the MLPA trials on the proband (II-3) and her son (III-2), her sister (II-1), and her niece (III-1) were normal. This demonstrated that the gene deletion was absent in the MEN1 of this family (Fig. 3).

A Chinese family presenting with MEN1, with a heterozygous mutation c.825-1G>A and a mutation in intron 5 (IVS 5-1 G>A) of the MEN1 gene, was considered by Zhiwei Ning et al. (13). Considering the possibility of an intron change, we redesigned the primer (NM_130802-3F, NM_130802-3R) in the middle of exon 2 and redetected with the WES test. It turned out that the proband, her sister, and her niece had heterozygous genetic mutations (c.201delC, p.Ala68Profs*51) in exon 2 of chromosome 11 (chr11:64577381), which is consistent with Mendelian genetic law (Fig. 2, MEN1-Exon2-3F, 3R).

\section{Discussion}

In this study, we found a proband with typical tumor symptoms of MEN1, including a pancreatic tumor, parathyroid tumor, adrenal tumor, and a suspicious gastrinoma, as well as pituitary changes. Subsequently, WES was used to test the proband for the MEN1 gene. We found a new gene mutation in exon 2 of MEN1, which could lead to these clinical symptoms. Due to the heritability of MEN1, the proband's family members were tested for MEN1 by Sanger sequencing. Subsequently, we found that this Chinese family had MEN1 and we found a new germline mutation of NM_130802 [c.201delC (p.Ala68Profs*51) on Chr11:64577381 on assembly GRCh37].

Because of the results from the genetic testing, we recommended that the proband's family members be hospitalized for screening for disease related to MEN1. Only the proband's sister (51 years old) accepted our recommendation for hospitalization. After several examinations, we found that the proband's sister also had the typical tumor symptoms of MEN1, including a pancreatic tumor and a parathyroid tumor. The proband and her sister's clinical symptoms indicated a MEN1 gene mutation in this Chinese family. However, due to a force majeure factor, the niece of the proband (III-1) rejected the recommendation to be hospitalized for screening the disease related to MEN1. In addition, any clinical symptoms related to MEN1 had not appeared. It may be because the MEN1-related tumor was not serious or she was not old enough. Therefore, we recommended that she be hospitalized at her earliest convenience for screening for the disease related to MEN1.

As we all know, the MEN1 gene plays a role as a growth suppressor in MEN1 tumorigenesis. In the germline, an inactivated mutation in the MEN1 gene on chromosome 11 causes MEN1 syndrome. About $90 \%$ of MEN 1 cases are usually inherited from affected parents; the other $10 \%$ of cases are due to a de novo mutation (9). The loss of heterozygotes at the MENIN site on chromosome 11q13 showed biallelic inactivation of MEN1 (14). Knudson's two-hit model of MEN1 gene tumorigenesis $(2,3,15)$ is supported by the harmful germline mutations observed in the MEN1 kindreds and the loss of wild-type alleles observed in the tumors of MEN1 patients in a MEN1 family. Since the cloning of the MEN1 gene in 1997, 
more than 1,000 families have been reported as MEN1 (16). Marini et al. reported an analysis of MEN1 mutations in 410 patients' germlines. It was found that there were 99 different mutations, of which 41 were frameshift, 26 missense, 13 nonsense, 11 splice site mutations, 4 in-frame small deletions, and 4 large intragenic deletions across one exon (17). In 2008, Lemos and Thakker conducted a comprehensive analysis of 1336 MEN1 mutations that had been reported in the first decade after the identification of the MEN1 gene (16). Paola Concolino et al. reported 208 new germline variants of MEN1 from 2007-2015 (18). No other similar papers were available in the literature apart from these MEN1 genetic mutationrelated data. We found the MEN1 genetic mutation c.201delC (p.Ala68Profs ${ }^{\star 51)}$ to be a new germline mutation site in exon 2 that had not yet been reported.

Interestingly, a very rare situation in this family is that the proband has the genetic mutation in exon 2 , and that intron 1 changed in the meantime. By coincidence, the primer region that we designed for WES was consistent with the changed intron 1 region (MEN1-Exon2-1F, 2F), which led to a homozygous mutation of MEN1 in the genetic detection results that could not be explained by Mendelian law of inheritance.

In the MEN1 gene, the most mutated exons are 2, 9, and 10. In particular, in exons 2 and 10, the most common type of mutation is a frameshift (18). Most of the frameshift and nonsense mutations cause protein truncation, resulting in the loss of functional domains, including NLSs located at the C-terminal segment. In the past few years, six new intron variants have been found in MEN1 patients. One of the intron variants (IVS3+18C>T) relates to a c.1546-1547insC mutation in a Chinese MEN1 family, which was reported by Zha et al. (19). Zhiwei Ning et al. (13) found a germline mutation of a heterozygous $G$ to A variation at the nucleotide position-1 of intron 5 (c.825-1G>A or IVS5-1G>A) in a MEN1 family. In this study, because of the change in intron 1 , homozygous mutations were found twice by WES. Therefore, we have reason to believe that intron 1 was also mutated. However, the new intron nucleotide mutation could be a polymorphism, and since introns have limited meaning in the structure of translation products, the mutations in the intron were not detected.

Gross deletions, usually detected by the MLPA technique (20-23), are the rarest kind of MEN1 mutation. The complete MEN1 gene deletion has been considered by different authors $(20,24,25)$. The first (exons $1-3)(22,23)$, central (exons 5-6) (21), and final (exons 8-10) regions of the gene (26) have been described by other gross deletions. Beijers et al. (12) were the first to report on a family with combined germline and somatic mosaicism for MEN1. They used MLPA to analyze the father and found both germline and somatic mosaicism of MEN1. Hence, it is necessary to use MLPA in MEN1 families who are difficult to diagnose. In this study, the MLPA results confirm that there were no significant gross deletions in the MEN1 gene in this family.

\section{Conclusion}

This is the first report of a Chinese family with a new MEN1 germline mutation in exon 2 (c.201delC (p.Ala68Profs*51)). It can improve the identification of clinical forms of MEN1, and can be used to 
diagnose the disease at an early stage. In addition, when it is difficult to diagnose the etiology, it is necessary to use MLPA to detect MEN1 and to consider SNP changes in introns, which have major implications for gene detection, treatment, and familial screening.

\section{Limitations}

The proband has temporarily refused surgery for any of her tumors, so we could not obtain tumor tissue for further genetic heterozygosity testing, pathological diagnosis, or cell biology testing. Because of the new intron nucleotide mutation may be a polymorphism, and introns have limited meaning to the structure of translation products, we did not further detect the mutations in the intron.

\section{Declarations}

\section{Ethics approval and consent to participate}

This study was performed following the approval of the institutional review board at Medical Science Research Ethics Committee of the First Hospital of China Medical University and the 1964 Helsinki declaration and its later amendments or comparable ethical standards.

\section{Consent for publication}

Informed consent was obtained from all individual participants included in the study. All the individual participants consented to the publication of medical data (including figures from diagnostic imaging results and from histological examination results). Patient's husband signs informed consent on behalf of all individual participants. All authors read and approved the final manuscript for publication.

\section{Availability of data and materials}

Data sharing is not applicable to this article as no datasets were generated or analyzed during the current study.

\section{Competing interests}

All author declare that they have no conflict of interest.

\section{Funding}

This study was supported by the Chinese National Natural Science Foundation (Grant: 81500605). The funder had no role in study design, data collection or analysis, or in the presentation or publication of the results.

\section{Authors' Contributions}


All the authors materially participated in this work, have read and approved the final manuscript. FZ is the first authors for this study. $X Y$ is the corresponding author supervising this work. FZ and $X L$ managed the case and drafted the manuscript. XW provides relevant ideas for research methods. FZ performed analysis on all data interpretation from literature review. XY reviewed the manuscript. We acknowledge that all authors participated sufficiently in the work and take public responsibility for its content.

\section{Acknowledgments}

Not Applicable.

\section{Authors' Information:}

Corresponding author's:

Xiaohui Yu, M.D., Ph.D., Professor, Department of Endocrinology and Metabolism, Institute of Endocrinology, Liaoning Provincial Key Laboratory of Endocrine Diseases, The First Affiliated Hospital of China Medical University, No. 155 Nanjing North Street, Heping District, Shenyang, Liaoning 110001, China. yuxh_1978@126.com, ORCID: 0000-0003-4802-9673

Telephone: +86 138-9880-9270

Other authors' E-mail addresses:

Fan Zhang, M.D., zhf935@163.com

Xinyue Lin, MBBS, yolanda.lin319@outlook.com

Xiaoli Wang, M.D., Ph.D., wlittlepear@163.com

All authors' ORCID:

ORCID number: Fan Zhang, (0000-0002-0176-2715); Xinyue Lin, (0000-0002-8168-7531); Xiaoli Wang, (0000-0002-9388-0734); Xiaohui Yu, (0000-0003-4802-9673)

\section{Abbreviations}

Multiple endocrine neoplasia type 1 (MEN1)

activator protein 1 (AP1)

loss of heterozygosity ( $\mathrm{LOH})$

whole exon sequencing (WES)

CT (Computed Tomography) 
MRI (magnetic resonance imaging)

endoscopic ultrasound (EUS)

parathyroid ECT (Emission Computed Tomography)

ethylenediaminetetraacetic acid (EDTA)

ACMG (American College of Medical Genetics and Genomics)

Multiplex Ligation-dependent Probe Amplification (MLPA)

insulin (INI)

C peptide (CPS)

pro-insulin (pro-INS)

oral glucose tolerance test (OGTT)

calcium $\left(\mathrm{Ca}^{2+}\right)$

phosphorus $(P)$

99mTc-stabimidine (MIBI)

cortisone (COR)

1-mg overnight dexamethasone (DXM)

prolactin (PRL)

growth hormone $(\mathrm{GH})$

IGF-1 (insulin like growth factor 1)

luteinizing hormone (LH)

follicle-stimulating hormone (FSH)

thyroid-stimulating hormone (TSH)

free-thyroxine (FT4)

neuro-endocrine tumors (NET)

gastrin 17 (GAS-17) 
Zollinger-Ellison Syndrome (ZES)

Entero-Chromaffin-Like (ECL)

hyperparathyroidism (HPT)

\section{References}

1. Brandi ML, Gagel RF, Angeli A, Bilezikian JP, Beck-Peccoz P, Bordi C, et al. Guidelines for diagnosis and therapy of MEN type 1 and type 2. J Clin Endocrinol Metab. 2001;86(12):5658-71.

2. Chandrasekharappa SC, Guru SC, Manickam P, Olufemi SE, Collins FS, Emmert-Buck MR, et al. Positional cloning of the gene for multiple endocrine neoplasia-type 1. Science. 1997;276(5311):4047.

3. Lemmens I, Van de Ven WJ, Kas K, Zhang CX, Giraud S, Wautot V, et al. Identification of the multiple endocrine neoplasia type 1 (MEN1) gene. The European Consortium on MEN1. Hum Mol Genet. 1997;6(7):1177-83.

4. Larsson C, Skogseid B, Oberg K, Nakamura Y, Nordenskjold M. Multiple endocrine neoplasia type 1 gene maps to chromosome 11 and is lost in insulinoma. Nature. 1988;332(6159):85-7.

5. Agarwal SK, Guru SC, Heppner C, Erdos MR, Collins RM, Park SY, et al. Menin interacts with the AP1 transcription factor JunD and represses JunD-activated transcription. Cell. 1999;96(1):143-52.

6. Thakker RV. Multiple endocrine neoplasia type 1 (MEN1). Best Pract Res Clin Endocrinol Metab. 2010;24(3):355-70.

7. Agarwal SK. Multiple endocrine neoplasia type 1. Front Horm Res. 2013;41:1-15.

8. van Asselt SJ, Brouwers AH, van Dullemen HM, van der Jagt EJ, Bongaerts AH, Kema IP, et al. EUS is superior for detection of pancreatic lesions compared with standard imaging in patients with multiple endocrine neoplasia type 1. Gastrointest Endosc. 2015;81(1):159-67 e2.

9. Singh G, Jialal I. Multiple Endocrine Neoplasia Type 1 (MEN I, Wermer Syndrome). StatPearls. Treasure Island (FL)2020.

10. Gatta-Cherifi B, Chabre O, Murat A, Niccoli P, Cardot-Bauters C, Rohmer V, et al. Adrenal involvement in MEN1. Analysis of 715 cases from the Groupe d'etude des Tumeurs Endocrines database. Eur J Endocrinol. 2012;166(2):269-79.

11. Norton JA, Krampitz G, Jensen RT. Multiple Endocrine Neoplasia: Genetics and Clinical Management. Surg Oncol Clin N Am. 2015;24(4):795-832.

12. Beijers H, Stikkelbroeck NML, Mensenkamp AR, Pfundt R, van der Luijt RB, Timmers $H$, et al. Germline and somatic mosaicism in a family with multiple endocrine neoplasia type 1 (MEN1) syndrome. Eur J Endocrinol. 2019;180(2):K15-K9.

13. Ning Z, Wang 0 , Meng $X$, Xing X, Xia W, Jiang Y, et al. MEN1 c.8251G>A mutation in a family with multiple endocrine neoplasia type 1: A case report. Mol Med Rep. 2015;12(4):6152-6. 
14. Marx SJ. Recent Topics Around Multiple Endocrine Neoplasia Type 1. J Clin Endocrinol Metab. 2018;103(4):1296-301.

15. Agarwal SK, Kester MB, Debelenko LV, Heppner C, Emmert-Buck MR, Skarulis MC, et al. Germline mutations of the MEN1 gene in familial multiple endocrine neoplasia type 1 and related states. Hum Mol Genet. 1997;6(7):1169-75.

16. Lemos MC, Thakker RV. Multiple endocrine neoplasia type 1 (MEN1): analysis of 1336 mutations reported in the first decade following identification of the gene. Hum Mutat. 2008;29(1):22-32.

17. Marini F, Giusti F, Fossi C, Cioppi F, Cianferotti L, Masi L, et al. Multiple endocrine neoplasia type 1: analysis of germline MEN1 mutations in the Italian multicenter MEN1 patient database. Endocrine. 2018;62(1):215-33.

18. Concolino P, Costella A, Capoluongo E. Multiple endocrine neoplasia type 1 (MEN1): An update of 208 new germline variants reported in the last nine years. Cancer Genet. 2016;209(1-2):36-41.

19. Zha BB, Liang W, Liu J, Cheng J, Hong XW, Liu J, et al. Mutation analysis in a Chinese family with multiple endocrine neoplasia type 1. Chin Med J (Engl). 2010;123(5):569-73.

20. Tham E, Grandell U, Lindgren E, Toss G, Skogseid B, Nordenskjold M. Clinical testing for mutations in the MEN1 gene in Sweden: a report on 200 unrelated cases. J Clin Endocrinol Metab. 2007;92(9):3389-95.

21. Owens $M$, Ellard $S$, Vaidya B. Analysis of gross deletions in the MEN1 gene in patients with multiple endocrine neoplasia type 1. Clin Endocrinol (Oxf). 2008;68(3):350-4.

22. Raef H, Zou M, Baitei EY, Al-Rijjal RA, Kaya N, Al-Hamed M, et al. A novel deletion of the MEN1 gene in a large family of multiple endocrine neoplasia type 1 (MEN1) with aggressive phenotype. Clin Endocrinol (Oxf). 2011;75(6):791-800.

23. Zatelli MC, Tagliati F, Di Ruvo M, Castermans E, Cavazzini L, Daly AF, et al. Deletion of exons 1-3 of the MEN1 gene in a large Italian family causes the loss of menin expression. Fam Cancer. 2014;13(2):273-80.

24. Mohrmann I, Gillessen-Kaesbach G, Siebert R, Caliebe A, Hellenbroich Y. A de novo 0.57 Mb microdeletion in chromosome 11q13.1 in a patient with speech problems, autistic traits, dysmorphic features and multiple endocrine neoplasia type 1. Eur J Med Genet. 2011;54(4):e461-4.

25. Rusconi D, Valtorta E, Rodeschini O, Giardino D, Lorenzo I, Predieri B, et al. Combined characterization of a pituitary adenoma and a subcutaneous lipoma in a MEN1 patient with a whole gene deletion. Cancer Genet. 2011;204(6):309-15.

26. Manzoni MF, Franchi GM, Sartorio SM, Di Rosa E, Cappelletti C, Piani C, et al. A novel MEN1 gene mutation. Cancer Genet Cytogenet. 2008;180(2):165-7.

\section{Tables}

Table 1. The laboratory examination results of hypoglycemia occured. 


\begin{tabular}{|lll|}
\hline Items & Results & Normal range \\
\hline & & \\
\hline Hypoglycemia occured for the first time & & \\
\hline Intravenous glucose $(\mathrm{mmol} / \mathrm{l})$ & 2.63 & $3.89-6.38$ \\
\hline Synchronous insulin $(\mathrm{mIU} / \mathrm{L})$ & 25.86 & $4.03-23.46$ \\
\hline Synchronous c-peptide $(\mathrm{pmol} / \mathrm{L})$ & 2052.1 & $99.9-1242.09$ \\
\hline Synchronous proinsulin $(\mathrm{pg} / \mathrm{mL})$ & 253.10 & $30-180$ \\
\hline Insulin release index & 0.55 & $<0.3$ \\
\hline Hypoglycemia occured for the second time & & \\
\hline Intravenous glucose $(\mathrm{mmol} / \mathrm{l})$ & 1.72 & $3.89-6.38$ \\
\hline Synchronous insulin $(\mathrm{mIU} / \mathrm{L})$ & 16.21 & $4.03-23.46$ \\
\hline Synchronous c-peptide $(\mathrm{pmol} / \mathrm{L})$ & 1994 & $99.9-1242.09$ \\
\hline Synchronous proinsulin $(\mathrm{pg} / \mathrm{mL})$ & 264.1 & $30-180$ \\
\hline Insulin release index & 0.52 & $<0.3$ \\
\hline
\end{tabular}

Table 2. The results of prolonged OGTT. 


\begin{tabular}{|llll|}
\hline & Glucose $(\mathrm{mmol} / \mathrm{l})$ & \multicolumn{1}{c|}{ Insulin $(\mathrm{mlU} / \mathrm{L})$} & C-peptide $(\mathrm{pmol} / \mathrm{L})$ \\
& & $(4.03-23.46)$ & $(99.9-1242.09)$ \\
\hline & & & \\
\hline 0 min & 5.16 & 15.45 & 1480.8 \\
\hline $30 \mathrm{~min}$ & 10.49 & 231.3 & 10060.5 \\
\hline 60 min & 13.11 & 217.2 & 10294.6 \\
\hline $120 \mathrm{~min}$ & 6.57 & 41.31 & 7194.8 \\
\hline $180 \mathrm{~min}$ & 3.72 & 21.52 & 3098.6 \\
\hline $240 \mathrm{~min}$ & 2.01 & 17.54 & 2501 \\
\hline $300 \mathrm{~min}$ & 1.95 & 15.2 & 1855 \\
\hline & & & \\
\hline
\end{tabular}

Figures 

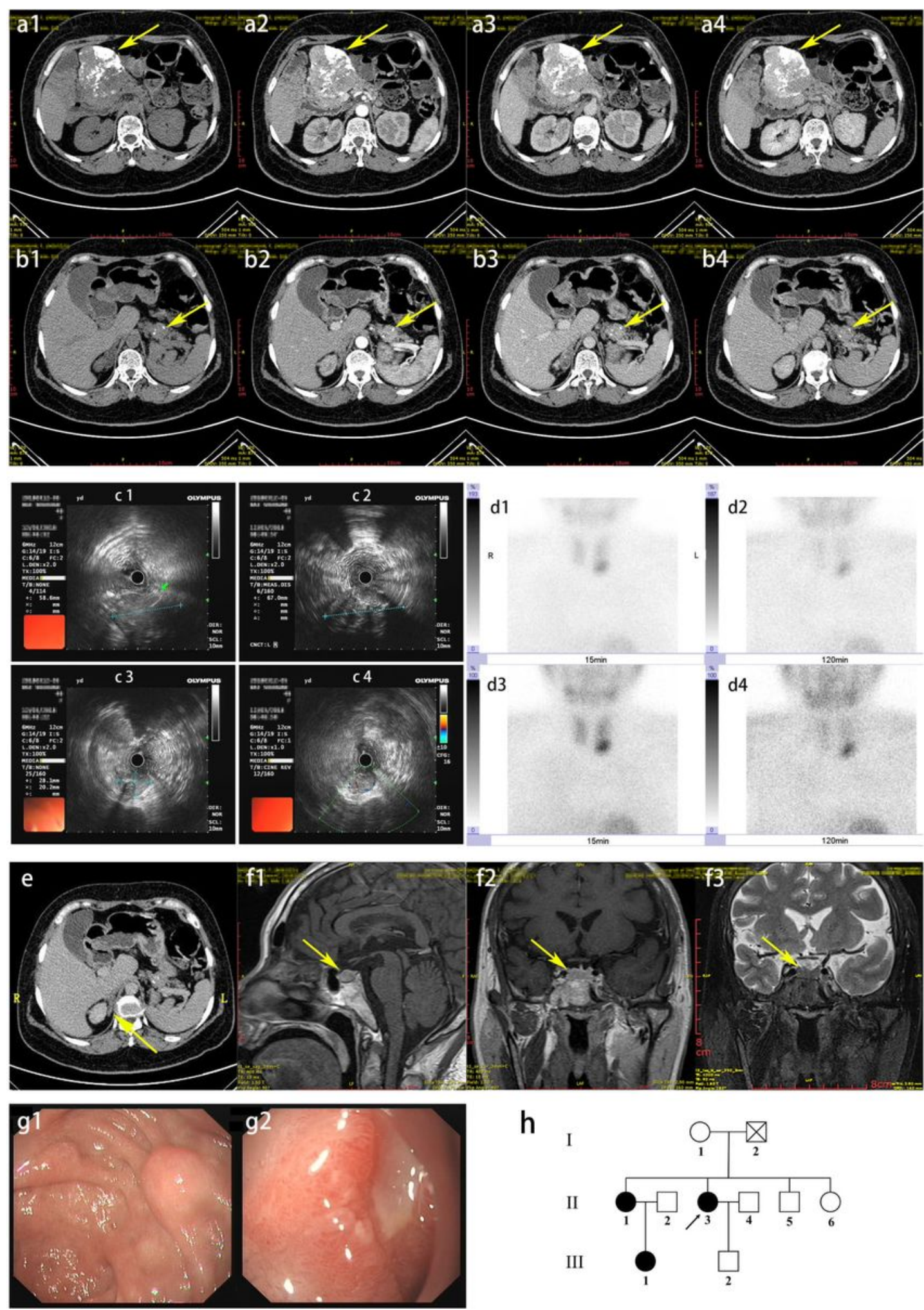

h

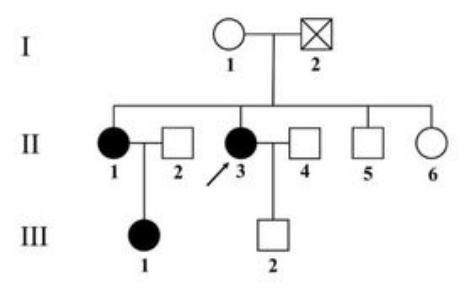

\section{Figure 1}

The proband's clinical imageology examination results. (a) a1-a4: Pancreatic neck lesions $(8.0 \times 6.8 \mathrm{x}$ $8.4 \mathrm{~cm}$ ) (a1, plain scan; a2, arterial phase; a3, portal phase; a4, delayed phase). (b) b1-b4: Ring enhanced nodule at the tail of the pancreas $(2.8 \times 2.7 \mathrm{~cm})$ (b1, plain scan; b2, arterial phase; b3, portal phase; b4, delayed phase). (c) The hypoechoic mass and the internal echoes were mixed. c1, c2: head of the pancreas (cannot be observed in the full field); c3, c4: the tail of the pancreas $(2.81 \times 2.02 \mathrm{~cm})$. (d) d1, d3: 
15 minutes after the $99 \mathrm{mTc}$-stabimidine (MIBI) injection, an area of increased imaging agent appeared in the lower lobe of the left thyroid; d2, d4: 2 hours after the injection, the area of distribution of the imaging agent under the left lobe of the thyroid did not decrease significantly. (e) Abdominal CT result showing that a mass occupied the right adrenal gland. (f) $f 1-f 3$. Pituitary-enhanced MRI examination showing that the hypophysis was full in shape (height $0.95 \mathrm{~cm}$ ). $\mathrm{f1}$, $\mathrm{f2}$ : T1Wl; $\mathrm{f3}$ : T2WI. $\mathrm{f} 1$ : sagittal view; $\mathrm{f2}, \mathrm{f3}$ : coronal view. (g) g1, Multiple submucosal uplifts (the larger was about $0.6 \times 0.8 \mathrm{~cm}$ ) at the junction of the duodenal bulb and the descending part of the duodenum; g2, the distal shallow ulcer in the descending part of the duodenum was about $1.0 \times 0.5 \mathrm{~cm}$ in size. (h) The patient's family consisted of three generations, including 10 members. The proband's father (I-2) of the first generation was deceased.

Seven people were tested, and the three members who carried the gene mutations were the proband (II-3), the proband's sister (II-1), and the proband's niece (III-1). 


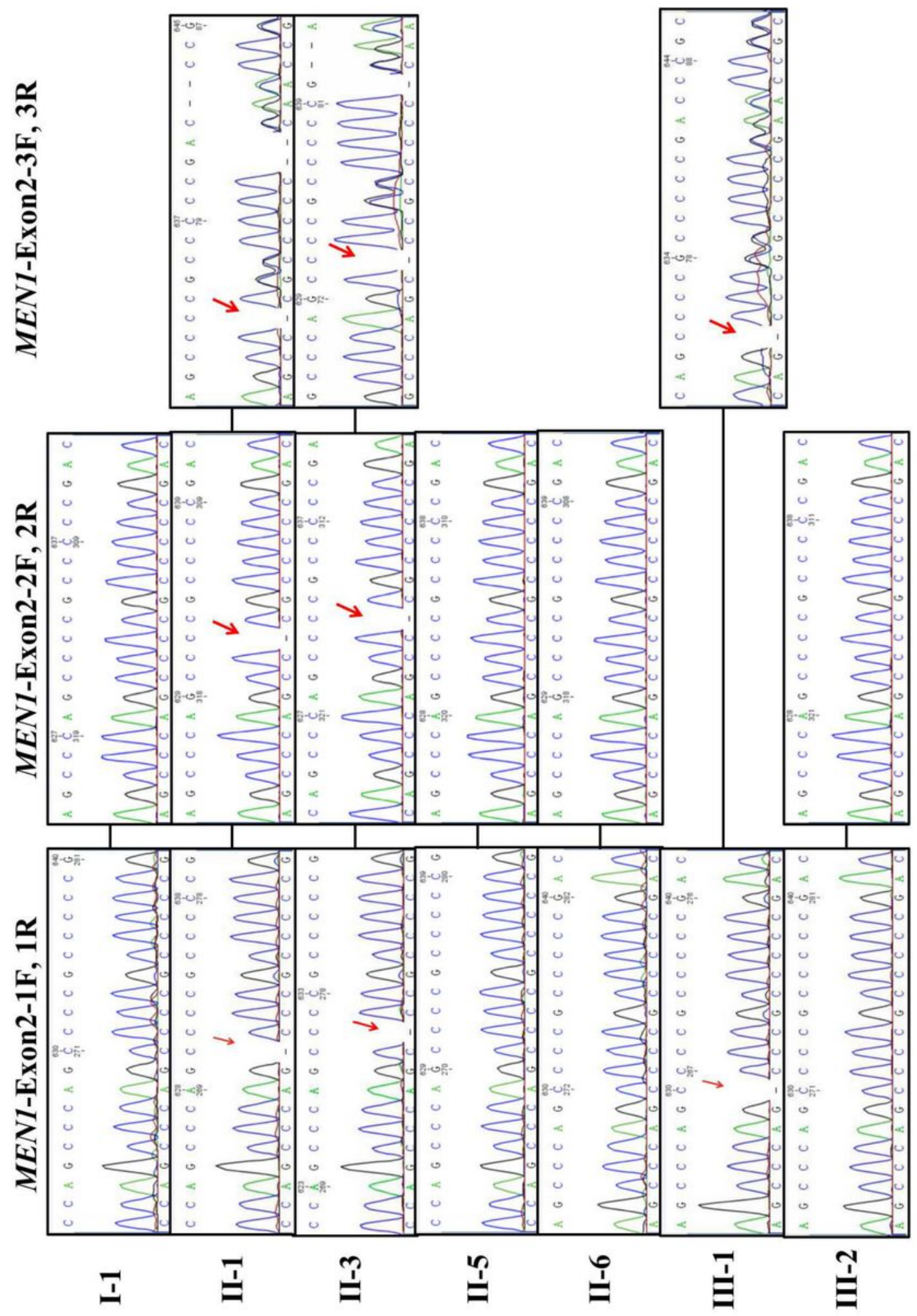

Figure 2

Whole exome sequencing results. The c.201delC change of NM_130802 in exon 2, which caused amino acid changes ( $p . A l a 68 P r o f s^{\star 51}$ ) that may have led to MEN1. The genetic changes were homozygous mutations (MEN1-Exon2-1F, 1R and 2F, 2R) and heterozygous mutations (MEN1-Exon2-3F, 3R). 


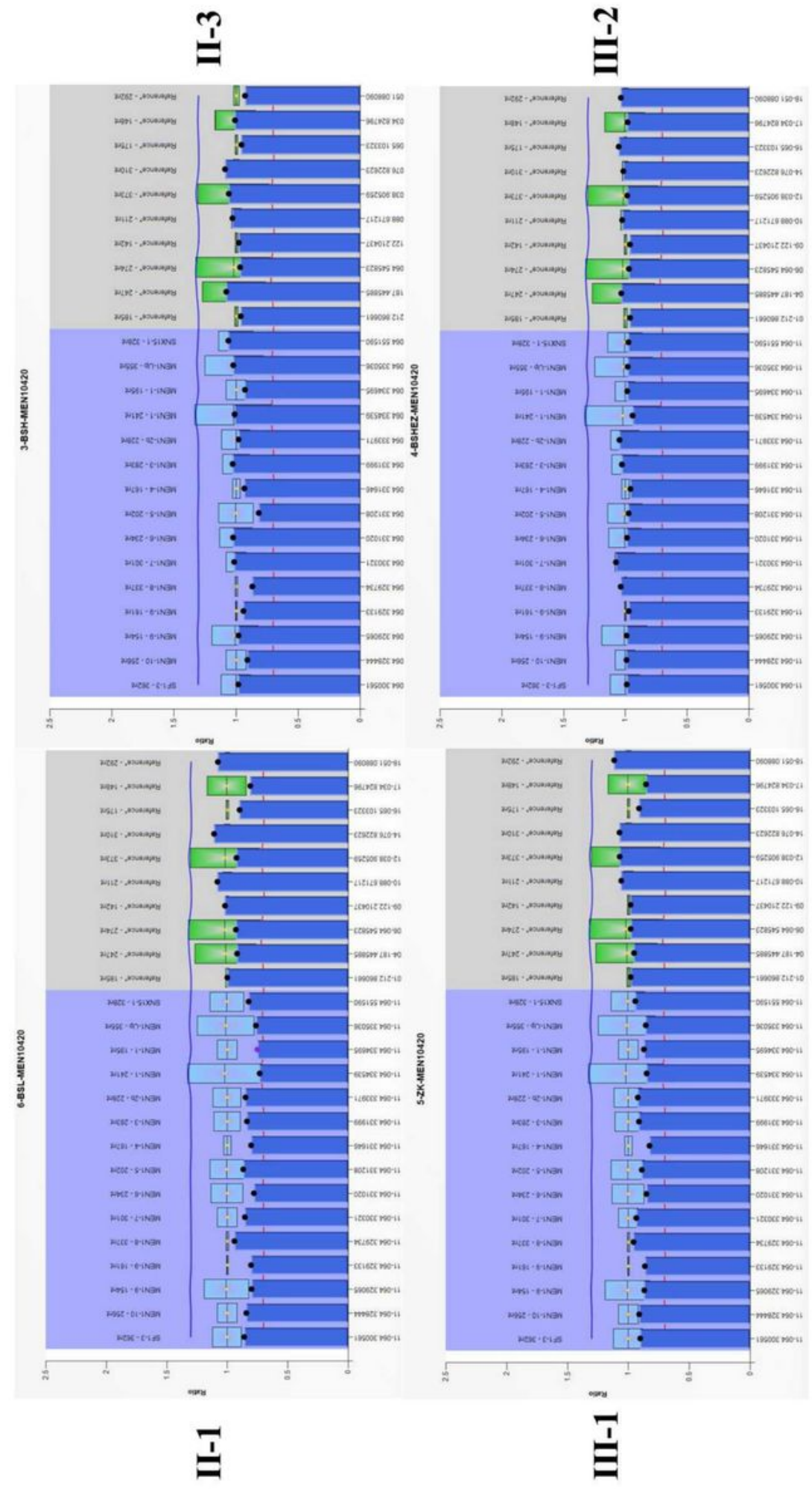

Figure 3

Multiplex ligation-dependent probe amplification (MLPA) results. MLPA results showed that the MEN1 gene was not deleted in the proband (II-3), the proband's sister (II-1), the proband's niece (III-1), and the proband's son (III-2). 